\title{
An Cognitive-IoE Approach to Ambient-intelligent Smart Home
}

\author{
Gopal Jamnal and Xiaodong Liu \\ School of Computing, Edinburgh Napier University, Edinburgh, UK \\ g.jamnal@napier.ac.uk,x.liu@napier.ac.uk,
}

Keywords: Intelligent inhabited environment, Ambient intelligent smart home, Activity pattern recognition, Cognitive IoTs, and Cyber Physical System.

Abstract:

In today's world, we are living in busy metropolitan cities and want our homes to be ambient intelligent enough towards our cognitive requirements for assisted living in smart space environment and an excellent smart home control system should not rely on the users' instructions. Cognitive IoE is a new state-of-art computing paradigm for interconnecting and controlling network objects in context-aware perception-action cycle for our cognitive needs. The interconnected objects (sensors, RFID, network objects etc.) behave as agents to learn, think and adapt situations according to dynamic contextual environment with no or minimum human intervention. One most important recent research problem is "how to recognize inhabitant activity patterns from the observed sensors data”. In this paper, we proposed a two level classification model named as ACM (Ambient Cognition Model) for inhabitant's activities pattern recognition, using Hidden Markov Model based probabilistic model and subtractive clustering classification method. While subtractive clustering separates similar activity states from non-similar activity states, a HMM works as the top layer to train systems for temporal-sequential activities to learn and predict inhabitant activity pattern proactively. The proposed ACM framework plays a significant role to identify user activity intention in more proactive manner such as routine, location, social activity intentions in smart home scenario. The experimental results have been performed on Matlab simulation to evaluate the efficiency and accuracy of proposed ACM model. 


\section{INTRODUCTION}

In recent years, IoT has envisioned the hardware technologies that let mobile and embedded devices to better exploit the web-internet features to connect anytime, anyplace, which enhanced the interactive experience of people centric Cognitive Internet of Things (Feng et al. 2017). As earlier claimed by Satyanarayanan (2001), great technology inventions are those that dissolve themselves into everyday life and be invisible for human consciousness. As a result, such research visions are making futuristic scenarios of Ambient Intelligence smart environments more promising into the reality of our everyday lives activities. The Cognitive IoT, overlaps the various research areas of pervasive computing, wireless-sensor networking, IOTs, artificial intelligence, machine learning and context-aware computing and cyber physical system. In CIoT, smart spaces extend the functionality of ambient intelligence toward more proactive possibilities, where smart environment not only monitors people for tasks, or support them by executing their requests, but also influences and changes their plans and intentions. Also by EU report, pervasive computing will be the next wave of new ICT innovation in the next five years, and it's said by 2020 it will be one major type of ICT system. Therefore CIoT has been viewed from the industry and the academic world as a main pillar of an upcoming industry revolution. The rapid use of interconnected network devices in healthcare systems, smart vehicles, transportation, classrooms, production units, smart homes, agriculture, would result a technological revolution in ubiquitous connectivity, computing and communication (Ricci et al. 2015 and Perera et al. 2014 and Feng et al. 2017).

In this paper our focus is on Cognitive IoT application in smart home for automated recognition of inhabitant activities to enhance the independent living experience to improve daily quality of life. In smart home scenario, obtained sensor data of inhabitant's activities interactions sequences within the environment is segmented and can be labeled as specific activity instance with description. The detected activity instances are used to train an activity recognition model as a result, the trained model are then used to classify and and assign a label to new activity instance. As Nazerfard et al. (2010) argued that discovering the order of activities can be effectively used for predicting the next activity in a home automation system using their temporal relation information. The activities pattern can be recognized by using machine-learning techniques such as HMM, Navies Bayes, Decision Tree, ANN and SVM and KNN etc. (Fahad et al. 2014 and Somov et al. 2013 and Wu et al. 2014).

The main aim of CIoT in smart home environment is to improve quality of life via developing an ambient intelligent living environment. The communication layer of multiple sensors can control home appliance via actuator/device controller to help inhabitant into the daily activities. In other words, CIoT works as brain, where raw data gathered from sensors and information collected and fused into decision making unit, for computing controlling commands to achieve specific goals. In such cases every home appliance can be programmed according to inhabitant need and living patterns. In industry, we have few smart solutions such as smart grids, electric meters, security controls system, lighting system, which can be programmed to customized as per individual desire (Feng et al.2017).

The research project aims to develop a novel dynamic architecture including its related models and mechanism to Cognitive-IoE based smart homes, where the functionality of ambient intelligence is extended towards more proactive possibilities, i.e., the smart environment not only monitors people/devices for tasks, or support them by executing their requests, but also influence and change their plans and intentions. In the dynamic environment, a home is equipped with multiple sensor (motion, light, noise) to perceive the environmental data in consistent/inconsistent state and preprocessed for further activity (similar/nonsimilar) classification. Human activity sequences can be analyzed from sensor data using their temporal values and transferred to an inference engine to recognized their daily activity patterns as routine, location and social contexts. 
The rest of this paper is organized as follows. Section 2 summaries related work on activity recognition done in the community. A bottom up approach to inhabitant activity recognition in smart space is presented as the ACM architecture in Section 3. The various classification based evaluation methods are presented in Section 4. The work is conclude in Section 5 and future work is discussed in Section 6. .

\section{SMART HOME: INHABITANT ACTIVITY RECOGNITION PROJECTS}

In recent years, learning and understanding the observed activity and event mining are the central research area to smart home studies. Activity refers to complex behaviors consisting of a sequence of action and overlapped action that can be performed by a single individual or several individuals interacting with each other. Some significant smart home activity recognition research work has been done in Care-lab, CASAS, Grator-Tech HIS, Aware Home, iDorm, and MavHom projects. In particular, the process of activity recognition can be divided in four steps such as i) sensing, ii) data-preprocessing, iii) data modelling feature extraction and iv) feature selection. The major research work is in progress by the tech giant IBM Watson, where cognitive appliances talk to each other and the central computing unit works as personalized digital assistant for granting access and controlling various appliances, example.g. smart locks, digital reminders, etc. IBM Watson's cognitive IoT vision is to create a custom tailored environment for individual residents by adapting their preference and patterns, which not only ensure better security, predictive maintenance tasks and alert system but also saves time and money of individuals, working as personal assistants (IBM Watson, 2017).

\subsection{SUBTRACTIVE CLUSTERING: PATTERN RECOGNITION IN SMART HOME}

While we talk about ambient intelligent smart home, the need of clustering methods arises to find relationships between observed datasets. As we know, clustering based classification is a well known approach to extract knowledge from obtained datasets by dividing datasets into discreet classified clusters. Two major clustering methods, K-means and the subtractive clustering can resolve the problem of separating similar and non-similar activities from given datasets (sensor's data). The kmeans clustering algorithms works on presegmented (known) clusters numbers, where we assume the number of clusters in advance for given datasets. Sometimes, it is not the case to have prior knowledge about the required number of clusters for obtained data sets. Also it makes system less flexible to identify appropriate cluster due to limited number of clusters. On the other hand, to improve the learning ability we can take the help of subtractive clustering algorithms to find patterns in inhabitant behaviors. As figure 1 shows, subtractive clustering methods estimate the cluster center and select the data point with highest potential value to be the first cluster center, and later remove all data points within the vicinity of the first cluster center in order to determine the next data cluster and its center location. The process keeps going on until all the remaining datasets identify their radius of a cluster center. Sub-clustering is a quick one pass algorithm, moreover it works in the context that the expert has no idea about the cluster number and specifications. In the same way, the smart home control system depends on the simulation of human experience to make itself an intelligent control system to perceptually make judgement to the external environment variables, which are very strong, moderate, and very weak (Amirjavid et al. 2014 and Fahad et al. 2014).

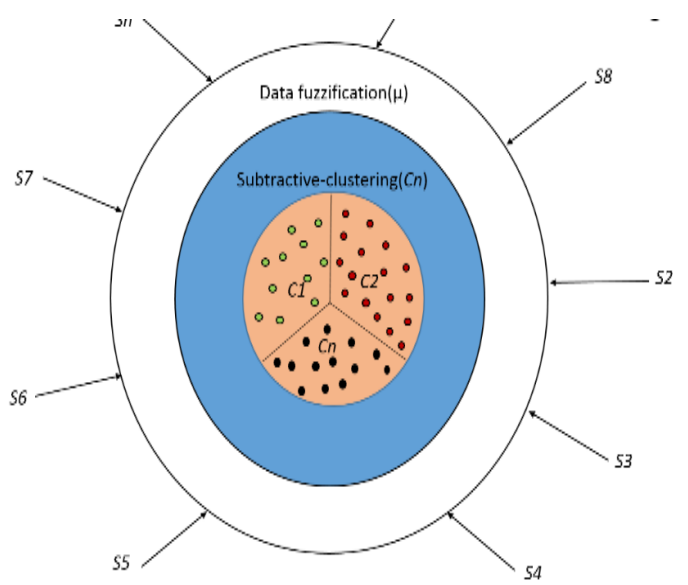

Figure 1. Subtractive clustering for data pre-processing 


\subsection{HIDDEN MARKOV MODEL: PATTERN RECOGNITION IN SMART HOME}

HMM is the most generative temporal probabilistic model, It is applied to find hidden states $(y 1, y 2, \ldots$. $, y T)$, in the observed time series data sequence $(x 1$, $x 2, \ldots, x T)$. HMM is fundamentally based on the independence of hidden states and the condition independence of observation parameters stipulating that $p(x t \mid y t, x 1, x 2 \ldots x t-1, y 1, y 2 \ldots y t-1)=$ $p(x t \mid y t)$. The observable state at time $t, x t$, depends only on the current hidden state $y t$. That is, the probability of observing $x t$ while being at $y t$ is independent of all other observable and hidden variables. The joint probability $p(x, y)$ of the observations and hidden states can be factorized as follows:

$$
p(x, y)=\prod_{t=1}^{T} p\left(y_{t} \mid y_{t-1}\right) p\left(x_{t} \mid y_{t}\right)
$$

The links between the hidden states are labeled with the transition probabilities and those between the hidden states and the observed sequence are labeled with the emission probabilities. Using the initial and transition probability matrix of the observable Markov model, it is possible to calculate the probability of any new activity sequences from the current activity sequence. That is the model can estimate the probabilities of new sequence, as shown in figure 2, HMM can solve three problems such as: Evaluation, Decoding and Learning problem. The evaluation problem uses a forward-backward algorithm to evaluate the probability of efficient computation based on a given set of observations. The learning problem optimizes parameters of a model to better describe the observations using the expectation-maximization (EM) algorithm. Finally, the decoding problem uses the Viterbi algorithm to find the most likely state sequence given an observation sequences. (Benmansour et al. 2016)

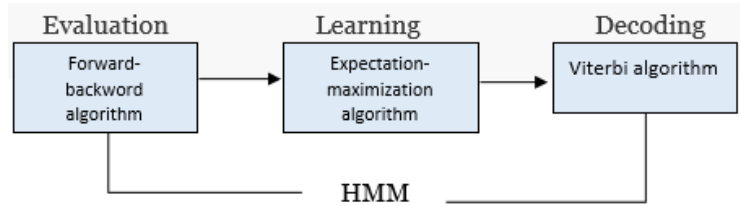

Figure2, Problem solving stages in HMM. (Benmansour et al. 2016)
As HMM jobs to identify the most likelihood sequences in given observed data vectors, so it is a bit challenging to adjust or re-estimate the model parameters to obtain the maximized probability of likelihood sequence vector. Therefore, an iterative approach of Baum-welch algorithm can be applied to re-estimate the HMM parameters sub-optimally, as expectation maximization(EM) method. Steps are :

(1) $\lambda_{0}=$ initial model

(2) compute new $\lambda$ based on $\lambda_{0}$ and given observation sequence $\vec{x}$

(3) if $\log \operatorname{Pr}(\vec{x} / y)-\log \operatorname{Pr}\left(\vec{x} / \lambda_{0}\right)<\Gamma$ stops, else set $\lambda_{0}=\lambda$ and repeat step (2)

Here, $\ulcorner$ represents the minimum tolerance value between two subsequent model.

Afterwards, the forward-backward algorithm places the re-estimated parameter in set of equations as below;

$$
\operatorname{Pr}(\vec{x} \mid \lambda)=\sum_{\forall Q} \operatorname{Pr}(\vec{x} \mid Q, \lambda) \operatorname{Pr}(Q \mid \lambda)
$$

Here, Q=states sequences (q1,q2, ..., qk)

$\lambda=$ HMM model

$\vec{x}=$ input data vector $<x 1, x 2, x 3 \ldots, \quad x k>$ of observation sequence. (Hassan et al. 2013)

\section{A BOTTOM UP APPROACH: ACM (AMBIENT COGNITION MODEL) FOR INHABITANT ACTIVITY RECOGNITION}

In particular, when we want to apply machine learning algorithms for activity pattern recognition in smart home scenario, we follow the sequential occurrence of regular activity in order to find changes in patterns of individual lifestyle. In addition, there are many methods for activity recognition in the related area, therefore it becomes important to consider general classification and examination of each approach and their implementation constraints for specific problem solution (Zolfaghari and Keyvanpour, 2016). In our research work, we applied the bottom-up approach for human activity recognition based on the datadriven probabilistic model. In contrast, the key 
contribution of our model is to identify user's key intentions based on observed sensors data. We used, subtractive clustering and Hidden Markov Model combination to recognize activities patterns in smart homes. As we applied the two level classification model in our proposed ACM framework in figure 3, inhabitant activity recognition is done in four stages; sensing, pre-processing, data modelling and context relevancy check such as social, environmental and activity intentions.

Figure 3. Proposed ACM (Ambient-Cognition

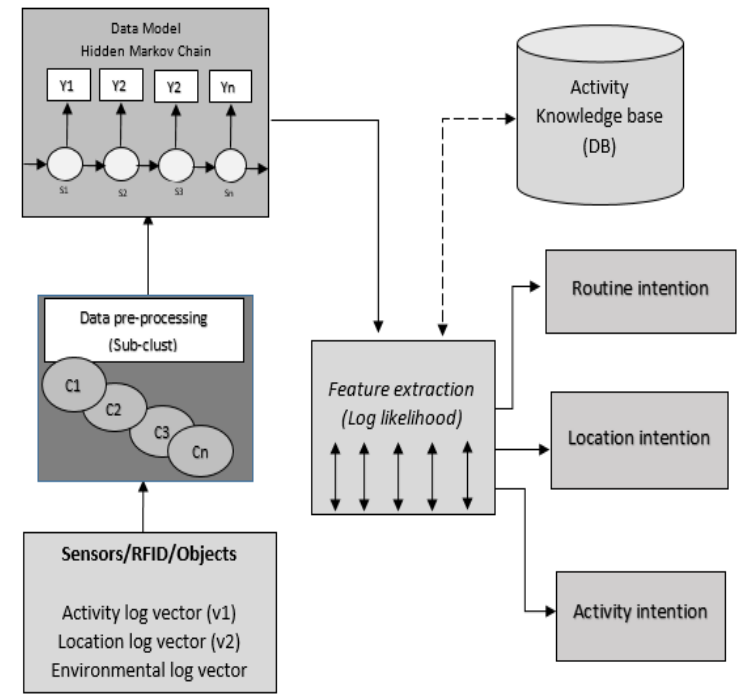

Model) framework components

In the first step, the sensor raw data are collected, containing information about objects, which have temporal information of each activity event. Furthermore, in order to separates the relationship between similar and non-similar activities, a subtractive clustering methods is applied to find relevant clusters in datasets. The sub-cluster algorithm works on radius parameter to identify the number of clusters. As a result, the large set of data problem is subdivided into the small datasets problem. Afterwards, using initial transition probability and emission matrix of the observed sequences, we applied Baum-welch algorithm to reestimate the HMM probability (transition and emission) matrix input parameters. later the ACM trained for 111 datasets using a re-estimated probability matrix.

We applied Viterbi algorithm to compute most likelihood sequence (probable-path) to find the maximum occurrence over all possible state sequences of given observed sequence datasets, which make our ACM model more ambient intelligent and proactive to understand inhabitant intentions. Furthermore, likelihood sequence is evaluated comparing to real time observed date sequence to check system accuracy and intelligence to identify recognized patterns. The following Viterbi equation can find most likely sequences of hidden states of given observations:

$$
\operatorname{argmax}_{x_{1} \ldots x_{t}} P\left(y_{1}, \ldots, y_{t}, y_{t+1} \mid x_{1: t+1}\right)
$$

Furthermore, we identify the user's intentions from HMM likelihood sequences, we can subdivide them into further three categories such as activity intention of users' movements for specific work, and location intention of user's specific movement and appearance on specific locations. In particular, a routine intention to identify user's daily activities can be outlined with repetitive task performed. Along with this, the activity knowledge base will be storing intentions for historical information purpose to link up for future reference.

\section{EVALUATION METHODS FOR ACM FRAMEWORK}

In an ambient environment, ADL (Activity daily living) can be evaluated in many different ways such as training and testing of each routine activity. Besides, there might be possibility of data scarcity of each activity, which might create problem of accuracy and performance evaluations. Above all, major problem might occur when inhabitant is reluctant to reveal their behavioral data due to privacy and ethical consideration. Therefore, a researcher should consider the availability of activities data sets before analyzing human activity recognition in ambient intelligent smart spaces.

For our research, we used existing ADL (activity daily living) datasets of multiple ubiquitous sensors placed in various location provided by MIT media Lab from Tapia (2003) research work. We will be using provided activity sequence of 111 samples of single user activity which are labeled based on each activity type.

Table 1, Activity daily living with their unique ID. 


\begin{tabular}{|c|c|}
\hline Activity Daily Living & ID \\
\hline Preparing dinner & 1 \\
\hline Watching TV & 2 \\
\hline Toileting & 3 \\
\hline Preparing breakfast & 4 \\
\hline Preparing lunch & 5 \\
\hline Dressing & 6 \\
\hline Taking medication & 7 \\
\hline bathing & 8 \\
\hline talking on phone & 9 \\
\hline cleaning & 10 \\
\hline
\end{tabular}

However, the main problem in MIT media lab data set is that, no time interval has been set in experimental data sets. As a result, number of activity states are not equal for each day. For example, some days have more activities sensors values compare to other days (day1 captured 5 activities, day2 have 9 activities captured). It could have been better if value 0 is provided where no activities event happened for specific day to maintain number of activity states in correct sequence manner. Hence, all days would have equal set of data value in matrix. In other words, every row has the same length. The below figure 4, represents the 10 activities (mentioned in table 1) of 111 observed sequences from MIT media lab during $19 / 4 / 2003$ to $31 / 4 / 2003$.

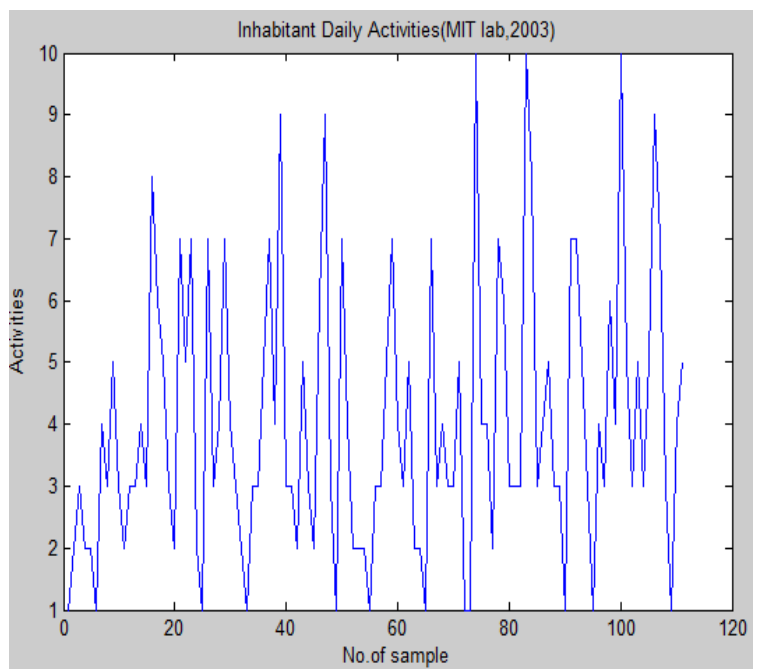

Figure 4, MIT media lab activity datasets
Furthermore, these 10 household activities are subclustered into 3 main activities clusters such as personal needs (1), domestic work (2) and relaxing (3) labels, mentioned in below table 2.

Table 2, three-activity cluster of household

\begin{tabular}{|c|c|}
\hline Activity Cluster & Type \\
\hline Personal needs(1) & Taking medication \\
\hline & bathing \\
\hline & talking on phone \\
\hline & Dressing \\
\hline Domestic work(2) & toileting \\
\hline & Preparing lunch \\
\hline & Preparing dinner \\
\hline & cleaning \\
\hline Relaxing(3) & preparing breakfast \\
\hline
\end{tabular}

Matlab 2016 version were used for test experiments, furthermore 111 observed activity datasets have been labeled into 3 main activities. Initial transition and emission matrix has been defined based on prior knowledge base. Afterwards, using Baum-welch algorithm further re-estimation of transition and emission probability matrix has been successfully achieved. Correspondingly, Matlab train the system for 111 observed sequences and Viterbi algorithm identified most likelihood activity sequences with the $77 \%$ of accuracy, shown in figure 5 as Matlab simulation result.

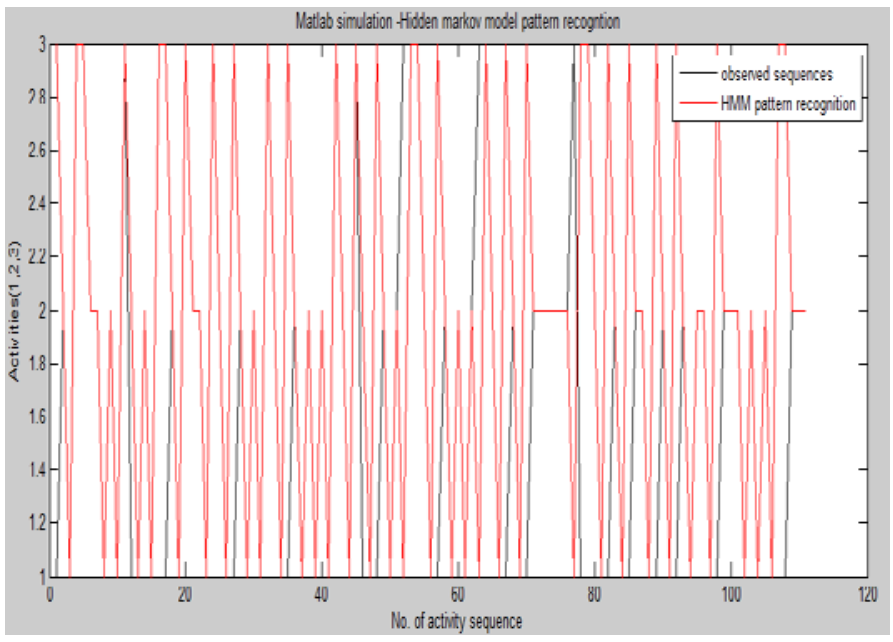

Figure 5. HMM likelihood activity sequence compares to observed sequence. 


\section{CONCLUTION}

This paper proposed a new type of ambient intelligent architecture for smart homes to understand the inhabitant intentions proactively, combining knowledge from traditional context aware pervasive systems with modern era Cognitive IoT technologies. The building block of ACM architecture is well equipped with artificial intelligence machine learning algorithms of subtractive clustering and Hidden Markov model to recognize patterns in daily activity living. ACM ensures the comfortable living environment for inhabitant as its primary goal. Moreover, the ACM model can be successfully applied in elderly health care systems, smart classrooms, and smart spaces for automated environment. While given MIT media lab data sets itself a detailed activities observation of inhabitants but could have been lot more better if have more data samples are available to avoid the data scarcity problem. In addition, the provided MIT media lab datasets could have better attributes in well-organized time set intervals. However, our ACM model well trained over provided data samples and successfully achieved $77 \%$ of likelihood sequence accuracy.

\section{FUTURE WORK}

As part of our ongoing work, we are extending our ACM to adapt a fuzzy-rule based system for executing tasks for physical world. We plan to combine ACM with device controllers by inferring fuzzy rule activations. Thus, it will be an excellent device controller for all appliances in smart homes/spaces. In addition, we would be creating our own smart home lab, where sensors data will be collected in predefined time set interval to maintain data accuracy and uniformity. As a result, the data scarcity problem would be avoided automatically and the result would be simulated with increased pattern recognition accuracy.

\section{REFERENCES}

Amirjavid, F., Bouzouane, A., \& Bouchard, B. (2014). Data driven modeling of the simultaneous activities in ambient environments. Journal of Ambient Intelligence and Humanized Computing, 5(5), 717740.

Benmansour, A., Bouchachia, A., \& Feham, M. (2016). Multioccupant activity recognition in pervasive smart home environments. ACM Computing Surveys (CSUR), 48(3), 34.

Fahad, L. G., Tahir, S. F., \& Rajarajan, M. (2014). Activity recognition in smart homes using clustering based classification.

Feng, S., Setoodeh, P., \& Haykin, S. (2017). Smart Home: Cognitive Interactive People-Centric Internet of Things. IEEE Communications Magazine, 55(2), 3439.

Hassan, M. R., Ramamohanarao, K., Kamruzzaman, J., Rahman, M., \& Hossain, M. M. (2013). A HMMbased adaptive fuzzy inference system for stock market forecasting. Neurocomputing, 104, 10-25.

IBM Watson, (2017) Watson Internet of Things [Online] https://www.ibm.com/internet-of-things/iot-zones/iotbuildings/cognitive-systems-in-buildings/ [accessed 14/01/2017].

MIT (2003) Using Machine Learning for Real-time Activity Recognition and Estimation of Energy Expenditure [Online] $<$ http://alumni.media.mit.edu/ emunguia/pdf/PhDThe sisMunguiaTapia08.pdf $>$ [Accessed 12/01/2017].

Munguia Tapia, E. (2003). Activity recognition in the home setting using simple and ubiquitous sensors (Doctoral dissertation, Massachusetts Institute of Technology).

Nazerfard, E., Rashidi, P., \& Cook, D. J. (2010, December). Discovering temporal features and relations of activity patterns. In 2010 IEEE International Conference on Data Mining Workshops (pp. 1069-1075). IEEE.

Perera, C., Liu, C. H., Jayawardena, S., \& Chen, M. (2014). A survey on internet of things from industrial market perspective. IEEE Access, 2, 1660-1679.

Ricci, A., Piunti, M., Tummolini, L., \& Castelfranchi, C. (2015). The Mirror World: Preparing for MixedReality Living. IEEE Pervasive Computing, (2), 6063.

Satyanarayanan, M. (2001). Pervasive computing: Vision and challenges.Personal Communications, IEEE, 8(4), 10-17.

Somov, A., Dupont, C., \& Giaffreda, R. (2013). Supporting Smart-city Mobility with Cognitive Internet of Things, 1-10.

Wu, Q., Ding, G., Xu, Y., Feng, S., Du, Z., Wang, J., \& Long, K. (2014). Cognitive Internet of Things: A New Paradigm beyond Connection. IEEE Internet of Things Journal, $\mathrm{PP}(99)$,

$1-1$. http://doi.org/10.1109/JIOT.2014.2311513.

Zolfaghari, S., \& Keyvanpour, M. R. (2016). SARF: Smart activity recognition framework in Ambient Assisted Living. In Computer Science and Information Systems (FedCSIS), 2016 Federated Conference on (pp. 1435-1443). IEEE. 